\title{
(5) $\mathfrak{e} \mathfrak{m} \mathfrak{h} \mathfrak{l} \mathfrak{d}$ \\ ber \\ Mievolutionen in Europa,

$$
\text { f } \in \mathbf{i} \mathbf{t}
$$

Dem Lmfturze Des Siỏmifdzen Raiferthums im

Decibent, bis auf unfre Deiten.

Mit ciner Einleitung in bie (S)efolichte, hronologifaen und genealogifdyen Tabellen, Randearten und einem yollfiandigen

'Siegiffer.

$\mathfrak{P} \cap \mathfrak{n}$

Chriftoph $\mathfrak{W}$ ilfelm Rod,

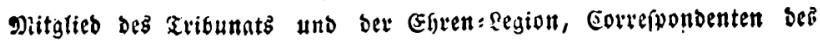
National : Эnttitutz.

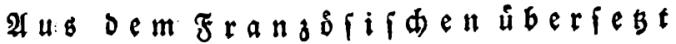

v 0 tt

3. D. $\subseteq$ $\mathfrak{a} \mathfrak{n}$ o $p \mathfrak{r}$

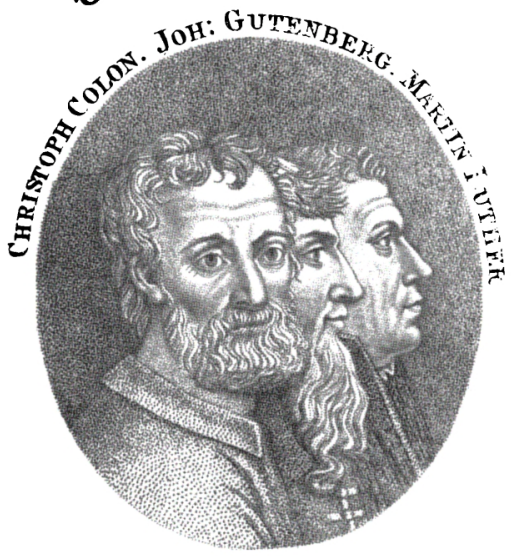

$\mathfrak{3}$ weiter $\mathfrak{z}$ a $\mathfrak{n}$ o.

Dait zwei und neunjig genealogifden Tabellen.

$$
\mathfrak{B} \mathfrak{e} \mathfrak{l} \mathfrak{i} \mathfrak{n} \text {, }
$$

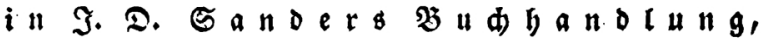
I 807 . 
